\title{
Schwerpunktthema: Psychiatrie und Psychotherapie im Strafvollzug
}

\author{
Elmar Habermeyer ${ }^{1}$ \\ Angenommen: 4. Oktober 2021 \\ (c) Springer-Verlag GmbH Deutschland, ein Teil von Springer Nature 2021
}

Bereits Heft 1 des Vorjahres befasste sich mit dem Strafvollzug und der Straftäterbehandlung, wobei der initiale Beitrag dieses Heftes vom Gefängnis als Entwicklungsraum sprach und für eine erweiterte Perspektive auf den Justizvollzug (Dahle et al. 2020) plädierte. Diesem Entwicklungsprozess wird in der Schweiz im Rahmen des Projekts Risikoorientierter Strafvollzug (ROS) Rechnung getragen. Daniel Treuthardt und Melanie Kröger aus Zürich berichten, aufbauend auf einem ersten Beitrag zum Thema (Treuthardt und Kröger 2021), der sich mit dem Projekt und seinen Grundprinzipien beschäftigte, nun über Möglichkeiten der Veränderungsmessung und über Rückfalldaten aus dem Projekt. Sie kommen zu dem Schluss, dass der Ansatz aus personen- und umweltbezogener Arbeit mit Straftäterinnen und Straftätern hilfreich ist und sehen ihre Resultate als einen Anstoß dafür, die Auswirkungen der Bearbeitung umweltbezogener Aspekte vertieft zu betrachten. Dass die Rückfälligkeit seit Einführung von ROS zurückgegangen ist, werten sie als Erfolg.

Hinsichtlich vollzugsbegleitender Behandlungen hat das Programm „Reasoning and Rehabilitation“ (R\&R; Ross et al. 1988) auch im Justizvollzug der deutschsprachigen Länder eine herausragende Bedeutung erlangt. Umso verwunderlicher ist der Mangel an Daten zur Effektivität dieses Programms in unserem Sprachgebiet. Daher ist es sehr zu begrüßen, dass Daniela Hosser und Sofia Weber aus Brauschweig eine Evaluation des Programmes im deutschen Justizvollzug vorstellen. Sie berichten anhand einer Stichprobe von 134 Häftlingen über positive Veränderungen der erfassten Risiko- und Schutzfaktoren mit konkreten Auswirkungen auf den Verlauf der Haft, denn Programmteilnehmer zeigten im Vergleich zur Kontrollgruppe weniger neue Regelverstöße.

Prof. Dr. med. Elmar Habermeyer

elmar.habermeyer@pukzh.ch

1 Klinik für Forensische Psychiatrie, Psychiatrische Universitätsklinik Zürich, Zürich, Schweiz
Der psychiatrische Beitrag des Journal-Club, erneut verfasst von Stjepan Ćurić aus Hamburg, befasst sich mit einer Studie von Beaudry et al. (2021), die hervorragend zum Schwerpunktthema passt und hinsichtlich der Effektivität von psychologischen Interventionen im Gefängnissetting eine skeptischere Position als die beiden vorab vorgestellten Arbeiten einnimmt. Es bedarf also weiterer Untersuchungen, und schon dieser Aspekt macht deutlich, dass uns das Schwerpunktthema auch in Zukunft beschäftigen wird und beschäftigen sollte.

Unabhängig von den vorgenannten, kriminalpräventiven Ansätzen ergibt sich eine weitere Herausforderung für den Vollzug dadurch, dass die Häftlingsklientel auch hinsichtlich der psychischen Gesundheit eine besonders belastete Gruppe darstellt. Dies verdeutlichten exemplarisch Zahlen von Von Schönfeld et al. (2006) und bezüglich der Situation in der Schweiz von Moschetti et al. (2015). Demnach ist in Häftlingspopulationen mit einem Anteil von 5-8\% psychotischer bzw. schizophrener Psychosen zu rechnen. Diese Gruppe von psychisch schwer kranken Gefangenen, die im Anlassverfahren, z. B. mangels Begutachtung oder weil sie keine Angaben gemacht haben bzw. effektiv dissimuliert haben, durch die Maschen gefallen ist, wird angesichts der zunehmend kritischen Haltung der Verteidiger gegenüber Begutachtungen zukünftig nicht kleiner werden.

Ein weiteres, für die medizinische Versorgung zunehmend relevantes Problem ergibt sich durch die zunehmende Anzahl älterer Häftlinge. Hierzu stellen Helene Seaward et al. aus Basel die Ergebnisse einer breit angelegten Schweizer Erhebung zu der psychischen Gesundheit und Versorgungssituation dieses Personenkreises vor. Die Resultate machen deutlich, dass die besonderen Bedürfnisse dieser mittlerweile am schnellsten wachsende Personengruppe in Haft bislang kaum erfasst sind, und dass hinsichtlich der Ausgestaltung altersgerechter medizinischer Versorgungssysteme und Lebensräume ein erheblicher Nachholbedarf besteht. Dass sich hieraus große therapeutische Herausforderungen auch für die Gefängnispsychiatrie und dort tätige Psychotherapeuten ergeben, haben Voß et al. für den Bereich der Sicherungsverwahrten und Langstrafler mit 
3 Fallvignetten bereits vor 10 Jahren prägnant dargestellt (Voß et al. 2011).

Die Bedeutung einer angemessen psychiatrisch psychotherapeutischen Versorgung unterstreicht auch der aus Covilhã, Portugal, eingereichte, englischsprachige Beitrag von Vitor Costa et al. Die Autoren befassen sich auf der Basis einer europäischen Studie damit, wie Personal und Häftlinge die gesundheitliche Verfassung der Häftlinge und die im Gefängniskontext verfügbaren Hilfsangebote wahrnehmen. Die Autoren plädieren darüber hinaus für eine intensive Schulung des Gefängnispersonals über psychische Störungen, was angesichts deren hoher Prävalenz (Opitz-Welke und Konrad 2021) in Haftpopulationen nur konsequent ist.

Unabhängig vom Schwerpunktthema reflektiert ein Beitrag von Mario Staller et al. aus Aachen das Thema der professionellen polizeilichen Kommunikation. Der Beitrag macht deutlich, mit welchen Herausforderungen man dabei sowohl in der Praxis als auch in der Ausbildung konfrontiert ist. Die Autoren definieren blinde Flecken, ein Ansatz der im Übrigen auch jeder anderen im forensischen Feld tätigen Berufsgruppe empfohlen werden kann, und plädieren, ähnlich wie es Lorey und Fegert (2021) für Kontakte zwischen Polizei und psychisch auffälligen Personen getan haben, für eine intensivere Schulung der Polizeibeamten. Die Auseinandersetzung mit methodischen Schwächen und der daraus resultierende Schulungsbedarf sind auch hinsichtlich der Befragung von einer Straftat verdächtigen Personen bedeutsam, was die Arbeit von Teresa Schneider und Lennart May aus Berlin verdeutlicht. Die Autoren befassen sich nämlich mit den Auswirkungen von Vernehmungstaktiken auf falsche Geständnisse und beleuchten die dahinterliegenden sozialpsychologischen Wirkmechanismen. Auch in dieser Arbeit wird für ein enges Zusammenwirken zwischen Psychologen und Polizei plädiert, um Fehlerquellen ausschalten zu können.

Weitere Beiträge befassen sich mit forensisch-psychiatrischen Maßregeln, wobei Adelheid Bezzel aus Regensburg, die in den vorherigen Ausgaben geführte Debatte (Schalast 2021; Müller 2021) um die Entwöhnungsmaßregel nach $\S 64$ StGB aufnimmt und durch ihre Daten zu den Verläufen einer forensischen Aufnahmestation bereichert. Ähnlich wie die Frage der Behandlungsabbrüche wegen Erfolglosigkeit im Rahmen von Entwöhnungsbehandlungen nach $\S 64$ StGB Fragen aufwerfen, sind auch die Erledigungen aus Gründen der Verhältnismäßigkeit im Maßregelvollzug nach $\S 63$ StGB in vielfältiger Weise interessant bzw. relevant für die forensische Psychiatrie. Schließlich stellen solche Erledigungen nicht das gewünschte Ergebnis einer Behandlung dar und sie erschweren oftmals auch das Übergangsmanagement zwischen Station und Nachsorge. Daher ist es sehr verdienstvoll, dass die Untersuchung von Nora Hein et al. aus Herne und Bochum Daten zu Merkmalen der laut ihren Resultaten heterogenen Patientengruppe lie- fert, die von solchen Verläufen/Entlassungsentscheiden betroffen ist. Der Schreibende ist schon jetzt gespannt auf die in der Arbeit angekündigten Untersuchungen zur Rückfälligkeit dieser Klientel.

Ergänzt werden die vorab erwähnten Übersichts- und Originalarbeiten durch das Blitzlicht, in dem sich HansLudwig Kröber kritisch mit der Umformulierung der Eingangskriterien der $\S 20$ bzw. $21 \mathrm{StGB}$ auseinandersetzt. Der kriminologische Beitrag von Marleen Gräber und Barbara Horten befasst sich mit dem Phänomen der Clankriminalität. Diese Form der organisierten Kriminalität hat in Deutschland innerhalb der letzten Jahre eine große öffentliche Beachtung gefunden, wobei beim Lesen oder beim Anschauen mancher journalistischer Beiträge der Eindruck entstand, dass eher ein Beitrag zur Selbstdarstellung der Protagonisten als zur Information geleistet wurde. Als Alternative empfiehlt sich die Lektüre des Beitrags aus Heidelberg.

Abschließend folgt der Kongresskalender, auf den der Autor dieser Zeilen diesmal besonders hinweisen will. Die wieder gewonnene Möglichkeit, Fachtagungen besuchen zu können, ist nicht nur wegen des persönlichen Austauschs zu begrüßen. Vielmehr leisten die im Kalender aufgeführten Veranstaltungen über die Möglichkeit zur lebhaften Diskussionen über Daten, die resultierenden Schlussfolgerungen und mögliche Konsequenzen für Behandlungskonzepte einen wertvollen Beitrag zur Weiterentwicklung unserer therapeutischen und kriminologischen Fächer. Die FPPK leistet dazu in schriftlicher Form einen Beitrag, den persönlichen Austausch kann und will sie aber nicht ersetzen.

Interessenkonflikt E. Habermeyer gibt an, dass kein Interessenkonflikt besteht.

\section{Literatur}

Beaudry G, Yu R, Perry AE, Fazel S (2021) Effectiveness of psychological interventions in prison to reduce recidivism: a systematic review and meta-analysis of randomised controlled trials. Lancet Psychiatry 8:759-773

Dahle KP, Greve W, Hosser D, Bliesener T (2020) Das Gefängnis als Entwicklungsraum. Ein Plädoyer für eine erweiterte Perspektive auf den Justizvollzug. FPPK 14:3-21

Lorey K, Fegert JM (2021) Polizeilicher Kontakt zu psychisch erkrankten Menschen. FPPK 15:239-247

Moschetti K, Stadelmann P, Wangmo T, Holly A, Bodenmann P, Wasserfallen JB, Gravier B et al (2015) Disease profiles of detainees in the Canton of Vaud in Switzerland: gender and age differences in substance abuse, mental health and chronic health conditions. BMC Public Health 15:1-11

Müller JL (2021) Replik zur Debatte um die Reform der Unterbringung in einer Entziehungsanstalt. Forens Psychiatr Psychol Kriminol 15:284-287. https://doi.org/10.1007/s11757-021-00665-w

Opitz-Welke A, Konrad N (2021) Psychiatrische Probleme im Justizvollzug. In: Dressing H, Habermeyer E (Hrsg) Psychiatrische Begutachtung, 7. Aufl. Urban \& Fischer, München, S 393-405 
Ross RR, Fabiano EA, Ewles CD (1988) Reasoning and rehabilitation. Int J Offender Ther Comp Criminol 32:29-35. https://doi.org/10. 1177/0306624X8803200104

Schalast N (2021) Zur Debatte um die Reform der gesetzlichen Voraussetzungen einer Unterbringung in der Entziehungsanstalt. Forens Psychiatr Psychol Kriminol 15:179-187. https://doi.org/10.1007/ s11757-021-00652

Von Schönfeld CE, Schneider F, Schröder T, Widmann B, Botthof U, Driessen M (2006) Prävalenz psychischer Störungen, Psychopathologie und Behandlungsbedarf bei weiblichen und männlichen Gefangenen. Nervenarzt 77:830-841
Treuthardt D, Kröger M (2021) Evaluation des Risikoorientierten Sanktionenvollzugs - Konstruktion einer Veränderungsmessung. Forens Psychiatr Psychol Kriminol 15:73-83. https://doi.org/10. 1007/s11757-020-00641-w

Voß T, Sauter J, Kröber HL (2011) Entlassene Problemfälle in der ambulanten Nachsorge von langzeitinhaftierten und langzeituntergebrachten Patienten. Forens Psychiatr Psychol Kriminol 5:253-260. https://doi.org/10.1007/s11757-011-0141-4 